\title{
GCU
}

Glasgow Caledonian

University

University for the Common Good

\section{Predictors of admission and readmission to hospital for major depression: A community cohort study of 52,990 individuals.}

Innes, Hamish; Lewsey, James; Smith, Daniel J

Published in:

Journal of Affective Disorders

DOI:

10.1016/j.jad.2015.04.019

Publication date:

2015

\section{Document Version}

Early version, also known as pre-print

Link to publication in ResearchOnline

Citation for published version (Harvard):

Innes, H, Lewsey, J \& Smith, DJ 2015, 'Predictors of admission and readmission to hospital for major

depression: A community cohort study of 52,990 individuals.', Journal of Affective Disorders, vol. 183, pp. 10-14. https://doi.org/10.1016/j.jad.2015.04.019

\section{General rights}

Copyright and moral rights for the publications made accessible in the public portal are retained by the authors and/or other copyright owners and it is a condition of accessing publications that users recognise and abide by the legal requirements associated with these rights.

Take down policy

If you believe that this document breaches copyright please view our takedown policy at https://edshare.gcu.ac.uk/id/eprint/5179 for details of how to contact us. 
TITLE: Predictors of admission and readmission to hospital for major depression: a community cohort study of 52,990 individuals.

AUTHORS: Hamish Innes ${ }^{1}$, James Lewsey ${ }^{2}$, Daniel J Smith ${ }^{2}$.

AFFILIATIONS:

1: Glasgow Caledonian University; School of Health and Life Sciences, Glasgow UK.

2: University of Glasgow; Institute of Health and Wellbeing; Glasgow, UK

WORD COUNT (inc : introduction, methods, results and discussion) $=1,750$ 
ABSTRACT:

BACKGROUND: Our current knowledge about predictors of admission and re-admission to hospital as a result of major depressive disorder (MDD) is limited. Here we present a descriptive analysis of factors which are associated with MDD hospitalizations within a large population cohort.

METHODS: We linked participants of the Scottish Health Survey (SHS) to historical and prospective hospital admission data. We combined information from the SHS baseline interview and historical hospitalizations to define a range of exposure variables. The main outcomes of interest were: (1) first time admission for MDD occurring after the SHS interview; and (2) readmission for MDD. We used Cox regression to determine the association between each predictor and each outcome, after adjusting for age, gender and deprivation quintile.

RESULTS: 52,990 adult SHS participants were included. During a median follow-up of 4.5 years per participant, we observed 530 first-time admissions for MDD. A relatively wide range of factors encompassing social, individual health status, and lifestyle-related exposures - were associated with this outcome $(\mathrm{P}<0.05)$. Among the 530 participants exhibiting a de novo admission for MDD during follow-up, 118 were later re-admitted. Only older age (over 70) and a prior non-depression related psychiatric admission were associated with readmission for MDD.

LIMTATIONS: MDD was defined using records of International Classification of Disease hospital discharge codes rather than formal diagnostic assessments.

CONCLUSION: These findings have implications for mental health service organisation and delivery and should stimulate future research on predictive factors for admission and readmission in MDD 
Highlights:

(1) We identified predictors of first time hospitalisation for major depressive disorder (MDD); and MDD readmission thereafter.

(2) A relatively broad range of social, health-status and lifestyle-related factors are associated with de novo admission for MDD.

(3) Only older age (over 70) and a prior non-depression related psychiatric hospital episode were associated with readmission for MDD.

\section{INTRODUCTION:}

Major depressive disorder (MDD) affects at least $15 \%$ of the population and is associated with chronic lifelong disability and high healthcare costs, often because of admission and readmission to hospital [1][2]. Our current understanding of how social, individual health-status and lifestylerelated factors influence risk of admission and readmission in MDD is limited. This is an important question for research because it could inform the development of new approaches to preventing both new and recurrent hospitalization for MDD.

In the current study we use data from the large and representative Scottish Health Survey cohort [3] to describe the relationship between a comprehensive range of characteristics - encompassing demographic, social, lifestyle, and physical health factors - and first-time admission (and also subsequent readmission) for MDD. Our intention is to present a broad descriptive picture [4] that could inform future interventions aimed at preventing admission and readmission to hospital for episodes of MDD. 
METHODS:

\section{SCOTTISH HEALTH SURVEY DATASET:}

The Scottish Health Survey (SHS) was launched in 1995 to monitor health behaviours and health outcomes in the Scottish population [3]. Detail on the sampling methodology underpinning the SHS can be found in section 1.2 of the SHS 2008 technical report [5]. Participation in the SHS involves a comprehensive face-to-face interview covering a broad range of health-relevant domains. Survey acceptance rates have generally been high, albeit have fallen with time (81\% response in $1995 ; 76 \%$ response in 1998; 60\% in 2003; and 54-56\% in 2008- 2011). Individual survey records have been electronically linked to pre- and post-interview hospitalisation and mortality records. Therefore by these linkage data surveyed persons are effectively participants in a prospective cohort study.[3] For this analysis, we combined the seven distinct waves of the $\operatorname{SHS}(95,98,03,08,09,10$ and 11 survey years) into one dataset with a view to assessing predictors of hospitalisation for MDD.

\section{ELIGIBILITY CRITERIA:}

We included adults (defined as 16 years or older at the time of the survey interview) participating in the 1995-2011 SHS, who had not previously been hospitalised for a MDD (as per the definition below).

\section{OUTCOMES OF INTEREST:}

1. Primary outcome: The primary outcome was a first-time hospital admission for MDD. Hospital admission data were taken from the SMR-01 and SMR-04 national Scottish databases (see [6] for detailed description). We used the presence of an F32 or F33 ICD-10 code in either the main or supplementary position to signify an MDD-related admission. We further performed a sensitivity analysis whereby MDD was defined according to the main diagnostic position only. To note, the ICD code present in the main diagnostic position indicates the principle condition the patient is being treated for, whilst the supplementary codes signify active comorbidities that have contributed to the overall episode.

2. Secondary outcome: For those individuals experiencing the outcome defined in 1, we further examined the occurrence of a depression-related readmission(where readmission was defined as per the same ICD codes listed above).

SOCIAL, LIFESTYLE AND HEALTH-STATUS PREDICTORS:

Summary data were available on twenty-two distinct social, lifestyle and health-status factors in total; as follows: 
(i) Social factors: age, gender, marital status, religious faith, sexual orientation, ethnicity, income quintile, employment in the last month, residential deprivation score, residential urbanicity.

(ii) General health status: life satisfaction, general health questionnaire score, Charlson comorbidity index[7], prior psychiatric hospitalisation (for a non depression-related cause), hospitalisation in the 12 months prior to survey, presence of a long standing illness.

(i) Lifestyle-related: i.e. physical exercise, consumption of fruit and vegetables, smoking status, weekly alcohol consumption, 'CAGE' alcohol use screening test, and body mass index (BMI).

The predictors above were mostly obtained from the baseline SHS interview, except for the Charlson Commorbidity index $(\mathrm{CCl})$, hospitalisation in the 12 months prior to survey, and previous hospitalisation for a psychiatric (but non-depression-related) cause. We instead derived these variables using historical hospitalisation data dating back to $1 / 1 / 1980$. We defined a psychiatric cause as the presence of an ICD-9 code: 290-319, or ICD-10 code: F00-F99 in any diagnostic position of the SMR-01 or SMR-04 databases. The CCI reflects both the extent and severity of diagnosed health conditions. It is calculated by assigning a score of 1-6, for each distinct co-morbidity present (with a higher score denoting greater severity). The total $\mathrm{CCl}$ score represents the sum of all individual comorbidity scores occurring prior to the survey interview. The presence or absence of each comorbidity was determined using the ICD 9/10 codes outlined by Quan et al [8].

\section{STATISTICAL ANALYSIS:}

\section{PREDICTORS OF DE-NOVO ADMISSION:}

We used Cox regression to describe the association between each predictor and the subsequent risk of a depression-related hospitalisation. For each participant, we commenced follow-up at the interview date and ended follow-up at the earliest instance of de-novo admission (if at all), or the censor date. The censor date applied was the date of death (if at all), or Dec-2012 (whichever came first). Hazard ratios are presented in their adjusted format (adjustment for: age, sex and residential deprivation quintile only). Unadjusted hazard ratios were also generated but due to space constraints are not presented in our main tables

\section{PREDICTORS OF READMISSION:}

Similarly, we used Cox regression to describe the association between each predictor and the subsequent risk of readmission for a depression-related cause. For each participant, follow-up began at the date of discharge from the index depression-related admission, and ended at the earliest instance of readmission (if at all), or the censor date. It is worth noting that although the start point 
of our readmission analysis is the index depression-related hospital episode; all the predictor variables examined in relation to a readmission event were collected at the baseline survey interview. As per our de novo admission analysis, hazard ratios presented in our results tables are adjusted for age, sex and residential deprivation quintile. 
RESULTS:

DESCRIPTION OF FINAL COHORT FOR DE-NOVO ADMISSION ANALYSIS:

We identified 52,990 adult participants of the SHS who had not previously been admitted to hospital for a depression-related cause. In total, these 52,990 individuals accrued 405,749 person-years (PY) of follow-up, during which time 530 first-time hospitalisations for MDD were observed (equating to a crude incidence rate of 1.3 per $1000 \mathrm{PYs})$. The mean follow-up time per participant from survey interview until outcome/censoring was 7.7yrs (median 4.5yrs).

\section{DERIVATION OF FINAL COHORT FOR READMISSION ANALYSIS:}

Of the 530 individuals admitted to hospital for MDD, nine died in connection to that hospitalisation, and one individual was discharged after the censor date. Thus we conducted our readmission analysis on the remaining 520 individuals. The total follow-up time amassed by these 520 individuals between their index hospitalisation and their censor date was 2,271 person-years. Over that time period, 118 individuals were readmitted, equating to a readmission rate of 52.0 per 1000 PYs. The mean follow-up time per readmitted participant was 4.4yrs (median 2.8yrs).

PREDICTORS OF DE-NOVO MDD ADMISSION (see Tables 1i, 2i, 3i):

In adjusted analyses, the following predictors were associated (at the $p<0.05$ level) with an increased risk of de-novo admission for MDD: older age (70+ years); female gender; unmarried relationship status; non-heterosexual sexual orientation; lower income; not working in the last month; living in a more deprived area; lower life satisfaction; higher general-health-questionnaire score; increased $\mathrm{CCl}$; previous admission for a psychiatric cause (other than MDD); hospitalisation in the 12 months prior to survey; presence of a long standing illness; fewer hours spent exercising; fewer portions of fruit and vegetables eaten; being a current smoker; drinking $>50$ units alcohol per week; and scoring two or more on the CAGE questionnaire. When a stricter definition of MDD, based on the main discharge code only was adopted (see eTables 1-3), most associations did not change appreciably. One exception was age $>70 y r s$, which in this sensitivity analysis, was no longer associated with newonset MDD.

PREDICTORS OF READMISSION FOR MDD (see Table 1ii, 2ii, 3ii):

In adjusted analyses, only older age-group (70+ years) and previous admission for psychiatric cause were significantly associated (at the $p<0.05$ level) with an increased risk of readmission for MDD. 


\section{DISCUSSION}

Our goal was to use routine health service data, linked to a large population-based cohort, to describe the impact of a wide range of social, lifestyle and health-related factors on hospital admission and subsequent readmission with MDD. We found that a relatively large number and diverse range of factors are associated with de novo admission in our adjusted analyses. Social factors in particular (such as gender, marital status, sexual orientation, income quintile, not working in the last month, and deprivation quintile) appear to be influential in the occurrence of MDD, which is consistent with previous studies [9-12]. The corollary of this observation is that population-level outcomes for MDD might be best improved through broad-based societal interventions, as opposed to individual-level approaches $[13,14]$. In contrast to our de novo admission analysis, we found that only two factors were associated with readmission, namely older age-group (70+ years) and previous admission for a psychiatric cause (other than MDD).

Significant strengths of this study include the very large sample size and representativeness of the Scottish Health Survey and the use of linked routine health service outcomes. However, some limitations are acknowledged. Despite assessing a large range of variables, we were limited in terms of the choice of variables available for this analysis by the assessments which were included in the baseline survey. Secondly, our readmission analysis has lower statistical power relative to our de novo analysis, and may be particularly prone to type-Il errors. Thirdly, for our readmission analysis, associations may be skewed towards the null due to regression dilution bias [15]. This is because we started follow-up at the date of index MDD admission, but derived exposure data from the survey interview that took place 6.0 years (on average) prior. Fourthly, for some predictors, missing data was substantial (more than $25 \%$ of participants were missing data for: religious faith; sexual orientation; income quintile; rural:urban indicator; fruit/vegetable consumption; smoking status and CAGE score ), but this was mostly due to these factors not being collected in the earlier waves of the survey, rather than any systematic non-response bias. Finally, the use of routine health service data may have limitations in terms of accuracy of the recorded discharge diagnoses, although we have been conservative in this regard by focussing on relatively narrow ICD groups.

In summary, within this large population cohort, we describe the influence of social, lifestyle and health-status relevant factors on the occurrence of MDD. These observational data represent an important body of evidence, that in conjunction with information from other scientific disciplines, could ultimately help us better understand the occurrence of MDD and the ways and means to control it. 


\section{REFERENCES:}

1. Collins, P.Y., Patel, V., Joestl, S.S., March, D., Insetl, T.R., Daar, A.S., Grand challenges in global mental health. Nature, 2011. 475: p. 27-30.

2. Whiteford, H.A., et al., Global burden of disease attributable to mental and substance use disorders: findings from the Global Burden of Disease Study 2010. The Lancet, (0).

3. Gray L, Batty GD, Craig P, Stewart C, Whyte B, Finlayson A, et al. Cohort profile: The Scottish health surveys cohort: linkage of study participants to routinely collected records for mortality, hospital discharge, cancer and offspring birth characteristics in three nationwide studies. Int J Epidemiol 2010. 39(2): 345-350.

4. Greenland S, Gago-Dominquez M, Castelao JE. The value of risk-factor ("black-box") epidemiology. Epidemiology 2004; 15:529-35.

5. The Scottish Health Survey 2008. Volume 2. Technical report. http://www.gov.scot/Resource/Doc/286063/0087159.pdf Accessed March 2015

6. Scottish National Health Databases: SMR-01: http://www.adls.ac.uk/nhs-scotland/generalacute-inpatient-day-case-smr01/?detail ; SMR-04: http://www.adls.ac.uk/nhsscotland/smr04-mental-health-inpatient-and-day-case-dataset/?detail ; $\mathrm{CHI}$ database: http://www.scot-ship.ac.uk/overview.html ; All accessed April 2014

7. Charlson ME, Pompei P, Ales KL, MacKenzie CR. A new method of classifying prognostic comorbidity in longitudinal studies: development and validation. J Chronic Dis 1987;40:37383.

8. Quan H, Sundararajan V, Halfon P, Fong A, Burnand B, Luthi JC, et al. Coding algorithms for defining comorbidities in ICD-9-CM and ICD-10 administrative data. Med Care. 2005;43:1130-9.

9. Moustgaard H, Joutsenniemi K, Martikainen P. Does hospital admission risk for depression vary across social groups? A population-based register study of 231,629 middle-aged Finns. Soc Psychiatry Psychiatr Epidemiol 2014; 49: 15-25.

10. Harris T, Cook DG, Victor C, Rink E, Mann AH, Shah S, et al. Predictors of depressive symptoms in older people - a survey of two general practice populations. Age Ageing 2003; $32: 510-8$

11. Warmer J, McKeown E, Griffin M, Johnson K, Ramsay A, Cort C, King M. Rates and predictors of mental illness in gay men, lesbians and bisexual men and women: Results from a survey based in England and Wales. Br J Psychiatry 2004; 185:479-85.

12. Wittayanukorn S, Qian J, Hansen RA. Prevalence of depressive symptoms and predictors of treatment among U.S. adults from 2005 to 2010. Gen Hosp Psychiatry 2014; 36:330-6. 
13. Link BG, Phelan J. Social conditions as fundamental causes of disease. J Health Soc Behav 1995; Spec No: 80-94.

14. Priebe s, Burns T, Craig TKJ. The future of academic psychiatry may be social. Br J Psychiatry. 2013;202:319-320.

15. Hutcheon JA, Chiolero A, Hanley JA. Random measurement error and regression dilution bias. BMJ 2010; 340: c2289 